Correction

\title{
Correction: Mas, S., et al. Identification and Chemical Characterization of Insoluble Contaminants in Hemodialysis Water Treatment. Water 2018, 10, 486
}

\author{
Sebastian Mas ${ }^{1,2, *(1)}$, Jesús Egido ${ }^{1,2,3}$, Alberto Ortiz ${ }^{1,3}$ (1) and Emilio Gonzalez-Parra ${ }^{1,3}$ (1) \\ 1 Renal, Vascular and Diabetes Laboratory, Fundacion Jimenez Diaz Research Institute (IIS-FJD), \\ 28040 Madrid, Spain; jegido@fjd.es (J.E.); AOrtiz@fjd.es (A.O.); EGParra@fjd.es (E.G.-P.) \\ 2 Spanish Biomedical Research Centre in Diabetes and Associated Metabolic Disorders (CIBERDEM), \\ 28029 Madrid, Spain \\ 3 Division of Nephrology and Hypertension, IIS-Fundación Jimenez Diaz UAM, 28040 Madrid, Spain \\ * Correspondence: smas@fjd.es; Tel.: +34-91-550-4800 (ext. 3292)
}

The authors regret omitting to explicitly mention some funds received when writing the original Acknowledgement Section [1]. The authors therefore wish to replace the Acknowledgments Section with the paragraph below:

Acknowledgments: The studies described above have been funded by the Spanish Ministry of Economy and Competitiveness and European Regional Development Funds (ERDF/FEDER) through ISCIII/FIS grants PI16/01298, PI17/01495, CIBERDEM and REDINREN RD016/0019 and through the Madrid Renal Society (SOMANE) grants. The authors thank the "Servicio Interdepartamental de Investigación" (Sidi) of the Universidad Autonoma de Madrid (UAM), and in particular Enrique Rodriguez.

The authors would like to apologize for any inconvenience caused to the readers by this change. The change does not affect the scientific results. The manuscript will be updated and the original will remain online on the article webpage, with a reference to this Correction.

\section{References}

1. Mas, S.; Jesús, E.; Ortiz, A.; Gonzalez-Parra, E. Identification and Chemical Characterization of Insoluble Contaminants in Hemodialysis Water Treatment. Water 2018, 10, 486. [CrossRef] 